\title{
O papel do agente comunitário de saúde: percepção de gestores municipais de saúde
}

\author{
The role of Community health workers: perception of municipal health mananger
}

El rol de los agentes comunitarios de salud: percepción de los gestores de salud municipales

Beatriz Santana Caçador ${ }^{1 *}$, Juliana Aparecida Caneschi ${ }^{1}$, Laylla Veridiana Castória Silva ${ }^{2}$, Ramon Augusto Ferreira de Souza ${ }^{1}$, Marilane de Oliveira Fani Amaro ${ }^{1}$, Lilian Cristina Rezende ${ }^{3}$, Carolina da Silva Caram³ ${ }^{3}$ Maria José Menezes Brito ${ }^{3}$.

\section{RESUMO}

Objetivo: Conhecer a percepção de gestores municipais de saúde sobre o papel do agente comunitário de saúde. Métodos: Estudo qualitativo, realizado em uma microrregião de saúde no interior de Minas Gerais. Participaram 8 secretários de saúde e 8 coordenadores da atenção primária à saúde. Coleta de dados ocorreu por meio de entrevista aberta orientada por roteiro semiestruturado, no período de abril a junho de 2018. Realizou-se Análise de Conteúdo de Bardin. Os aspectos éticos da pesquisa com seres humanos foram respeitados. Resultados: Os gestores reconhecem, de modo coerente com as atribuições previstas, o papel do agente comunitário de saúde, sobretudo, no que tange a constitução do elo entre a equipe de saúde e a comunidade, as facilidades de residir na comunidade e a dificuldade do reconhecimento de seu papel pela população. Além disso, apresentam-se conscientes sobre os desafios de deslocamento inscritos no cotidiano do agente comunitário de saúde, a falta de capacitação e de materiais de trabalho. Conclusão: Constata-se através do estudo, que os gestores reconhecem de modo coerente as atribuições previstas ao agente comunitário de saúde e afirmam reconhecer sua importância. Porém, essa valorização parece não encontrar eco nas condições de trabalho desse profissional viabilizadas pelos gestores.

Palavras-chave: Gestor de saúde, Agentes comunitários de saúde, Papel profissional, Enfermagem.

\begin{abstract}
Objective: Know the perception of municipal health managers about the role of the community health agent. Methods: Qualitative study carried out in a health microregion in the interior of Minas Gerais. Eight health secretaries and eight primary health care coordinators participated. Data collection took place through open interviews guided by a semi-structured script, from April to June 2018. Bardin's Content Analysis was performed. The ethical aspects of research with human beings were respected. Results: Managers consistently recognize the role of the community health workers, in a way that is coherent with their assigned duties, especially with regard to establishing the link between the health team and the community, the ease of living in the community and the difficulty of not having their role recognized by the population. They recognize the displacement challenges inscribed in the community health workers daily life, the lack of training and work materials. Conclusion: Managers recognize the role of the community health workers in a coherent way with the assignments that are foreseen for them and claim to recognize its importance. However, this appreciation does not seem to find an echo in the working conditions of the professional made possible by these managers.
\end{abstract}

Keywords: Health manager, Community health workers, Professional role, Nursing.

1 Universidade Federal de Viçosa (UFV), Viçosa - MG. *E-mail: cacadorbeatriz2@gmail.com

2 Universidade Federal do Espírito Santo (UFES), Vitória - ES.

3 Univerdidade Federal de Minas Gerais (UFMG), Belo Horizonte - MG. 


\section{RESUMEN}

Objetivo: conocer la percepción de los gestores de salud municipales sobre el rol del agente comunitario de salud. Métodos: Estudio cualitativo realizado en una microrregión de salud en el interior de Minas Gerais. Participaron ocho secretarias de salud y ocho coordinadores de atención primaria de salud. La recolección de datos se realizó a través de entrevistas abiertas guiadas por un guión semiestructurado, de abril a junio de 2018. Se realizó el Análisis de Contenido de Bardin. Se respetaron los aspectos éticos de la investigación con seres humanos. Resultados: Los gerentes reconocen consistentemente el rol del agente de salud comunitaria, de manera coherente con sus funciones asignadas, especialmente en lo que se refiere a la constitución del vínculo entre el equipo de salud y la comunidad, la facilidad para vivir en la comunidad y la comunidad. dificultad para que la comunidad no reconozca su papel. Reconocen los desafíos de desplazamiento inscritos en la vida diaria del agente comunitario de salud, la falta de formación y materiales de trabajo. Conclusión: A través del estudio, parece que los administradores reconocen consistentemente las atribuciones otorgadas al agente de salud de la comunidad y afirman reconocer su importancia. Sin embargo, esta valoración no parece encontrar eco en las condiciones laborales de este profesional posibilitadas por los directivos.

Palabras clave: Gestor de salud, Agentes comunitarios de salud, Rol profesional, Enfermería.

\section{INTRODUÇÃO}

A Constituição Federal Brasileira de 1988, associada à construção do Estado democrático de direito, possibilitou a saúde como um direito garantido a sociedade. É na Constituição que se encontram as leis orgânicas da saúde que estabelecem o Sistema Único de Saúde (SUS) como política de saúde no Brasil e a saúde como condição de cidadania de todo brasileiro. O SUS assume como princípios a equidade, universalidade e integralidade, e como diretrizes organizativas a descentralização, hierarquização, regionalização e participação social (MENEZES APR, et al., 2019).

Como forma de operacionalizar as mudanças advindas do SUS, criou-se a Estratégia Saúde da Família (ESF), compreendida como principal dispositivo para a organização do modelo de atenção proposto pelo SUS. A ESF possui como foco a família e, dessa forma, constitui-se como uma forma de reestruturar a atenção primária. Permeia-se à ESF a participação de toda a comunidade na formação do cuidado integral e holístico, em que a equipe de saúde atua em conjunto com a população (CAÇADOR BS, et al., 2015).

Neste contexto, há que se destacar a atuação do Agente Comunitário de Saúde (ACS), membro da equipe da ESF, cujas práticas possuem a singularidade de transitar entre o saber popular e o saber técnico da saúde. Isto porque o ACS é morador da comunidade e atua como um elo entre a equipe de saúde e a comunidade (BEZERRA YRN e FEITOSA MZS, 2019).

Tendo como prerrogativa a organização do SUS de forma descentralizada e, considerando a ESF como principal dispositivo de operacionalização do SUS, destaca-se o lugar do município nas políticas de saúde, por adquirir relevância e responsabilidade. O município passa, então, a ser o principal executor de ações de saúde, considerando a capacidade da governança local de eleger o que são as necessidades de saúde mais relevantes e sobre as quais precisa investir esforços e ações de saúde. Neste contexto, os gestores municipais de saúde tornam-se atores fundamentais para a tomada de decisão e organização das práticas e processo de trabalho de modo a tornar viável a consolidação do SUS no cotidiano das pessoas (MINISTÉRIO DA SAÚDE, 2009).

Neste sentido, considerando a importância da prática do ACS na consolidação do SUS no cotidiano das comunidades e, tendo como pressuspoto que a gestão muncipal é estratégica para delinear as práticas dos agentes comunitários de saúde, fortalecendo-as ou fragilizando-as, surge a inquietação: qual a percepção de gestores municipais de saúde sobre o papel do ACS?

A relevância do estudo circunstancia-se na governabilidade da gestão muncicipal de saúde na organização do processo de trabalho no contexto da saúde da família de modo que a função gerencial torna-se dispositivo 
estratégico para a viabilização de práticas e processos que sejam coerentes com os princípios do SUS. Mediante o exposto, o estudo possui objetivo de conhecer a percepção de gestores municipais de saúde sobre o papel do agente comunitário de saúde.

\section{MÉTODOS}

Trata-se de um estudo de natureza qualitativa por possibilitar ao pesquisador lidar com categorias explicativas e analíticas que extrapolam os dados quantitativos. Segundo Proetti S (2017) este caminho metodológico é coerente quando pretende-se compreender fenômenos subjetivos e analisar os fatos investigados em seu contexto.

O estudo foi realizado em uma microrregião de saúde do interior do estado de Minas Gerais, composta por dez municípios. Participaram do estudo oito coordenadores da atenção primária e oito secretários de saúde dos municípios em questão. Dois coordenadores e dois secretários não aceitaram participar do estudo. Os critérios de inclusão foram pertencer ao cargo de coordenador da atenção primária ou estar secretário de saúde no momento do estudo. Não houve critérios de exclusão.

A coleta de dados foi realizada no período de abril a junho de 2018 por meio de entrevista aberta orientada por roteiro semiestruturado. A entrevista aberta em pesquisa na saúde possibilita uma maior profundidade à reflexão do entrevistado, por isso a quantidade de material produzido é densa e profunda (MINAYO MCS e COSTA AP, 2018). Além disso, estabelece uma comunicação verbal dos indivíduos envolvidos na pesquisa no qual será imposto um fluxo de troca de saberes sem a estruturação total de questionamentos (FERREIRA VS, 2014).

O roteiro semiestruturado é estabelecido a partir de questões que o pesquisador supõe de acordo com o objetivo do estudo. A entrevista combina em questões abertas e fechadas de forma organizada, possibilitando ao entrevistado falar livremente sobre o tema proposto, de modo a facilitar ao entrevistador a capacidade de articular e dirigir o assunto (BONI V e QUARESMA SJ, 2005).

A entrevista aberta juntamente com o roteiro semiestruturado produzem um conjunto de dados com interesse pertinente, apresenta dinamicidade por deixar o entrevistado à vontade e se estabelece como um guia ao entrevistador durante a interlocução, contribuindo satisfatoriamente com a pesquisa (BONI $\mathrm{V}$, QUARESMA SJ, 2005; MINAYO MCS e COSTA AP, 2018).

Dessa forma, a participação do pesquisador foi ativa na condução e interação com o participante envolvido, sendo previamente treinado para tais. Foi realizado agendamento das entrevistas em horário e local previamente acordado com os participantes. Os depoimentos foram gravados e transcritos na íntegra, garantindo a fidedignidade dos dados. A fim de garantir sigilo os depoimentos dos coordenadores da atenção primária foram identificados em CA1 a CA8 e dos secretários de saúde em S1 a S8.

Os dados foram analisados por meio da técnica de análise de conteúdo proposta por Bardin L (2016), por permitir analisar as comunicações propostas entre o pesquisador e os sujeitos da pesquisa. Essa técnica envolve três polos: a pré-análise, a exploração do material e tratamento dos resultados e, por último, a inferência e interpretação. Os três passos foram seguidos a fim de garantir resultados adequados à luz da literatura.

A pesquisa trata-se de um recorte de um macroprojeto aprovado pelo comitê de ética (Parecer 1.135.193; CAAE: 44143615.2.0000.5153), a fim de respeitar os princípios éticos da pesquisa, em conformidade com a Resolução Conselho Nacional de Saúde 466/12, sobre Diretrizes e Normas Regulamentadoras de Pesquisa envolvendo Seres Humanos do Ministério da Saúde e Resolução 510 de 07 de abril de 2016, que dispõe sobre as normas aplicáveis a pesquisas em Ciências Humanas e Sociais, sendo observadas e aplicadas em todas as fases. Todos os direitos dos participantes foram assegurados garantindo a liberdade de recusar a participar ou retirar seu consentimento a qualquer tempo do estudo. Os participantes assinaram o Termo de Consentimento Livre e Esclarecido (TCLE) após serem informados sobre a garantia de anonimato, privacidade e utilização dos resultados somente para fins científicos. 


\section{RESULTADOS E DISCUSSÃO}

\section{A importância do fazer do Agente Comunitário de Saúde}

Evidencia-se com os depoimentos abaixo, que os gestores municipais possuem uma compreensão coerente com o real papel do agente comunitário de saúde, sobretudo, no que tange a constitução do elo entre a equipe de saúde e a comunidade. Além disso, o reconhecimentodo ACS como àquele que abre "portas" entre a familia que necessita ser assistida e a equipe para acolhê-la. Reconhecem, ainda, a importância desse papel para a resolutividade e efetivação da ESF.

"Então, o agente comunitário é a linha de frente, o elo do médico, do enfermeiro e até mesmo do técnico, que abre portas para que essa equipe chegue até aquela família" (S1).

"O agente comunitário de saúde tem papel fundamental na saúde do município, são eles, o elo entre a comunidade e à equipe" (CA2).

"O agente comunitário de saúde é aquele profissional importante dentro de uma equipe de saúde, é ele quem consegue criar e estender o elo entre a comunidade e os profissionais de saúde" (S3).

"Eu acho que na saúde da família é bem importante né, porque eles que vão nas casas [...] fazer visita em todas as casas pra olhar, pra acompanhar quem tem mais necessidade, tem que ir até mais vezes se precisar" (CA1).

"O ACS é o vínculo entre a ESF e a comunidade, ele tem a função de visitar as famílias e "levar saúde" até elas" (S7).

A aproximação do agente comunitário de saúde com a comunidade estabelece a formação de vínculos e criação de um elo entre a população e a unidade de saúde. As relações sociais entre o ACS e a comunidade ocorrem devido ao território ser considerado como um espaço vivo, no qual as pessoas demonstram suas singularidades, modos de conviver e arranjos socioculturais, a fim de enfrentarem a vulnerabilidade à que estão submetidas (BRASIL CCP, et al., 2021).

De acordo com a Politica Nacional de Atenção Básica (PNAB) de 2017, o ACS possui atribuições como: realizar visitas domiciliares às populações residentes em sua área sanitária, considerando os critérios de riscos e de vulnerabilidade das famílias; conhecer o funcionamento das ações e serviços do território, a fim de orientar a população quanto a utilização dos serviços de saúde disponíveis; estimular a comunidade à participar das políticas públicas voltadas à área da saúde, dentre outras. Portanto, a criação do elo entre a comunidade e o ACS, ocorre através do conhecimento das necessidades de saúde da população por meio de visitas domiciliares e orientações (MINISTÉRIO DA SAÚDE, 2017).

Além disso, os entrevistados também reconhecem como papel do ACS o desenvolvimento de práticas de promoção da saúde e prevenção de agravos, conforme evidenciado.

"O papel do agente é muito importante né,eles trabalham tanto na prevenção quanto na promoção da saúde, e eles são a porta de entrada do nosso serviço de saúde, porque eles que conhecem a realidade de cada família, conhecem o indivíduo, então são eles quetrazem pra gente todas às informações que tem na casa das pessoas, então assim é um elo muito importante entre a família das pessoas e as nossas unidades de saúde, então é de fundamental importância o papel deles" (CA4).

Ainda, segundo a PNAB todos os profissionais de saúde da atenção básica devem desenvolver estratégias de promoção da saúde e prevenção de doenças e agravos, entretanto, destaca que o agente comunitário de saúde por ser o elo entre a comunidade e a unidade de saúde, em especial, deve atuar ativamente no território por meio de visitas domiciliares regulares e de ações educativas individuais e coletivas, na ESF, no domicílio e outros espaços da comunidade (MINISTÉRIO DA SAÚDE, 2017). 
Assim, o ACS é considerado um personagem-chave na organização e planejamento da assistência prestada, pois é morador da comunidade e ao mesmo tempo parte da equipe de saúde. Nessa perspectiva, o ACS articula e media a relação entre os saberes científicos e os populares, com a finalidade de construir projetos de cuidados que atendam às especificidades de um determinado território. Ainda, o capital social produzido pelo ACS por ser membro/morador da comunidade no qual trabalha, potencializa a proximidade entre usuários e profissionais, o que possibilita ações de promoção da saúde e prevenção de agravos (SECCO AA, et al., 2021).

Dessa forma, de acordo com Jesus AS, et al. (2014) o vínculo entre o ACS e a comunidade é resultado de confiança e sustentado pelo acolhimento e cuidado. Por meio do vínculo construído e fortalecido no cotidiano das práticas do ACS e da proximidade que possuicom a realidade local, este torna-se facilitador do planejamento de ações de saúde, identificação dos riscos, danos e necessidades de saúde da população.

Por conhecer de modo singular a realidade das famílias que compõem a comunidade, o ACS torna-se fundamental para revelar os problemas dos usuários e possibilitar o cuidado integral, conforme as falas abaixo.

"O papel mais importante é o do ACS, são eles que trazem o problema do usuário até a coordenação, para resolver o problema. Sem o agente o serviço não flui” (S5).

"[...] é ele quem ajuda a sua equipe a conhecer melhor a comunidade, entender suas necessidades, criar estratégias para ofertar um serviço com qualidade" (S6).

"Eles têm um outro olhar, porque estão inseridos naquela comunidade e conhecem cada um dos usuários que acompanham" (CA2).

"Desde o inicio da fundação do SUS, o objetivo do ACS era chegar nas comunidades mais distantes e inserir o usuário na unidade de saúde" (S8).

Portanto, o ACS por residir no local onde está inserida a ESF e atuar como elo entre a população e a equipe de saúde possui facilidade em conhecer as necessidades de saúde da comunidade, monitorar casos e resultados de intervenções, promover facilidadede acesso à unidade e ao direito à saúde, realizar visitas aos domicílios, promover vigilância e ações de educação em saúde e realizar atividades com grupos prioritários e ações para agilizar o trabalho. Dessa forma, o ACS contribui para qualidade do cuidado a ser ofertado à população de maneira integral e humanizada (SAMUDIO JLP, et al., 2017).

Além disso, o fato de o ACS residir na comunidade em que trabalha, é tido como uma facilidade para o acesso à saúde e conhecimento profundo das necessidades do usuário, conforme os depoimentos abaixo.

"A maior facilidade é que quase todos moram na sua microárea, isso facilita o acesso" (S7).

"As facilidades do serviço do ACS pra mim é ele estar inserido numa comunidade onde ele já conhece, sabe bem das necessidades de cada um dos usuários" (CA2).

"Os ACS encontram como agente facilitador do seu trabalho a sua residência próximo aos usuários de sua microárea" (CA6).

Ademais, o ACS assume uma dupla inserção no seu meio relacional, destacando-se o fato de morar e trabalhar na comunidade os levando a terem duplo papel: o de ser agente e sujeito. Assim, além de estabelecer laços de identificação com o trabalho o ACS estabelece esses mesmos laços com comunidade mediante o vínculo, que em conformidade com suas atribuições, é sua ferramenta de trabalho e se exprime no encontro com o usuário (SANTOS AL, et al., 2017).

Por outro lado, essa aproximação entre o ACS trabalhador e o ACS morador, muitas vezes, causa dificuldade da população em reconhecer àquela pessoa como profissional, devido ao elo de amizade e, também, em compreender seu papel e suas atribuições, por cobrarem serviços que não estão ao alcance desses, conforme os depoimentos a seguir. 
"A maior dificuldade é a não compreensão do paciente sobre o trabalho do agente. O usuário cobra serviços que não são atribuições" (CA5).

"As pessoas conseguirem entender qual é o trabalho dos agentes, por que muitos confundem amizade com o trabalho que eles prestam e acabam por tratar eles mal" (S2).

Segundo Jesus AS, et al. (2014) o desconhecimento dos usuários sobre o trabalho do ACS implica em insatisfações com a atuação destes profissionais. O que propicia a reflexão sobre a importância de estratégias que esclareçam a atuação profissional do ACS para a comunidade.

\section{Os desafios na prática do agente comunitário}

A visita domiciliar é competênciado ACS, permeada por objetivos, como o contato ativo com as famílias e manutenção do elo entre a população e a ESF, permitindo desenvolvimento de estratégias que visem a prevenção de doenças e promoção da saúde, além da oferta de um cuidado ampliado. No entanto, algumas barreiras perfazem as visitas domiciliares, dificultando a sua prática (NASCIMENTO VL, et al., 2017). Dentre elas, destacam-se o acesso dificultado às residências, condições climáticas desfavoráveis, ambientes de visitação nem sempre agradáveis e seguros, falta de transportes para deslocamento, sobrecarga de serviço, dentre outros. Evidencia-se algumas dessas dificuldades através dos depoimentos a seguir.

"Dificuldade tem, por exemplo, tem agente de saúde que a área dele é bem extensa, aí então, tipo assim, dificuldades de locomoção"(S4).

"Eu acho que na zona rural tem uma dificuldade maior pela extensão, eles têm que andar muitos quilômetros pra poder chegar a essas famílias [...]cada um se locomove da sua maneira" (CA4).

Assim, devido a grande extensão da área em que o ACS é responsável, ocorre a necessidade de deslocamento de longas distâncias, e, em muitas vezes não há disponibilidade de carro de modo integral, o que dificulta a realização de continuidade do cuidado através das visitas domiciliares. Além disso, o ACS está exposto à fatores climáticos, como sol desgastante e chuvas. A exposição por horas sob à luz solar, se não ocorrer o uso de filtro solar de boa qualidade, pode causar manchas e/ou queimaduras, entretanto, esse e outros materiais como óculos protetores de raios ultravioletas e capa de chuva, geralmente são escassos (NASCIMENTO VL, et al., 2017).

Evidencia-se tais problemas por meio dos depoimentos abaixo, que relatam a falta de recursos físicos e materiais, inclusive de cadernos, canetas, borrachas, em que os próprios profissionais precisam comprar para conseguirem realizar seu trabalho.

"Falta de recursos: falta protetor solar, computador, transporte" (CA8).

"Falta de materiais necessários para realizar o seu trabalho, agente sabe que nem sempre tem disponível o protetor solar, canetas, lápis, borracha, caderno, às vezes eles acabam comprando" (S3).

"[...]a falta de alguns materiais, muitos deles tem que comprar a sua própria caneta, caderno" (CA2).

"O que eles falam às vezes é assim, calor com sol muito quente eles reclamam, que assim, eles têm essa dificuldade né" (CA1).

Além disso, nota-se mediante os depoimentos que o ACS não possui, com regularidade, capacitação e educação permanente.

"As dificuldades que tem hoje que a gente apontou foi a dificuldade da não capacitação que eles tiveram né [...] falta de educação permanente, porque é o seguinte, como eles entraram sem saber qual a função do agente, hoje nós estamos buscando isso pra eles, mostrando pra eles qual que é a função do agente" (S1). 


\section{"Mas o que mais eles reclamam é a capacitação mesmo, assim eles vêm falar pra ter mais capacitação" (CA1).}

Desta forma, por ser laço e vínculo da comunidade com a equipe de saúde, o ACS adquire por finalidade de trabalho atender indivíduos e famílias por meio de ações de monitoramento, visitas domiciliares e informação em saúde. Então, a fim de desempenhar essas funções o ACS necessita de capacitações e treinamentos adequados, que incluem desde procedimentos técnicos, até o desenvolvimento de atividades educativas e preventivas junto à população (BARBOSA VBA, et al., 2012).

Entretanto, em conformidade com outros estudos, nota-se que não há planejamento de educação permanente na ESF, além disso, as ações educativas são pontuais e aleatórias, muitas vezes desenvolvidas por agentes externos que destoam o processo de trabalho. Evidencia-se que não há um planejamento institucional de educação nas equipes da ESF, com um cronograma sistematizado de atividades. $O$ planejamento da educação não está sintonizado com os problemas enfrentados na realidade. A ação educativa não é coletivamente constituída, com a participação dos trabalhadores, gestores, serviços de saúde (MOROSINI MV e FONSECA AF, 2018).

Ademais, o trabalho do ACS favorece a democratização das informações, estimulação da participação da população na saúde, tanto no cuidado quanto nas políticas, e, por conviverem com diferentes pessoas e círculos, atuam ampliando o olhar para as práticas de saúde daqueles que mais necessitam. Entretanto, apesar do reconhecimento da centralidade do trabalho do ACS e seu importante papel na articulação entre comunidade e serviço de saúde, devido às tensões éticas vivenciadas em diferentes níveis, baixos salários e incentivos, o profissional tende a se sentir desvalorizado, o que compromete, muitas vezes, o andamento do serviço de qualidade na ESF (GUANAES-LORENZI C e PINHEIRO RL, 2016).

Neste sentido, a identidade social é entendida como um conjunto de qualidades que caracterizam o sujeito. Essas qualidades são entendidas como a essência do próprio ser, seus hábitos de vida, conhecimentos, costumes, formas de pensar e de se perceber no mundo. Portanto, a identidade social representa as transformações das características do indivíduo conforme suas relações sociais e deve ser entendida como um processo contínuo no qual o indivíduo e o julgamento que o outro faz irão sempre moldar essa identidade (DUBLAR C, 2012).

Assim, a perspectiva do entrevistado abaixo, demostra que há uma fragilidade na percepção de si por parte dos próprios trabalhadores que não reconhecem sua importância e, essa fragilidade de pertença de si, pode comprometer o desempenho de sua prática.

"É o mais importante de todos e só eles que não percebem isso. Eles se sentem desvalorizados, por isso o trabalho fica comprometido" (CA5).

Ademais, o estudo apresentou limitações como a necessidade de reagendar a data das entrevistas, mesmo que tenham sido previamente agendadas, devido diversos compromissos que os gestores possuem. Além disso, os gestores encontravam-se quase que em sua maioria apressados em decorrência de suas agendas de modo que não houve tempo de aprofundamento nas questões investigadas.

\section{CONCLUSÃO}

O estudo permitiu conhecer a percepção de gestores municipais sobre o papel do agente comunitário de saúde. Os gestores reconhecem o papel do ACS de modo coerente comas atribuições previstas pela política de saúde e afirmam reconhecer a importância da prática deste profissional. Porém, essa valorização parece não encontrar eco nas condições de trabalho do ACS viabilizadas pelos gestores os quais sinalizam desafios que poderiam por eles serem solucionados.

\section{REFERÊNCIAS}

1. BARBOSA VBA, et al. Educação permanente em saúde: uma estratégia para a formação dos agentes comunitários de saúde. Rev Gaúcha Enferm., Porto Alegre (RS), 2012; 33(1): 56-63. 
2. BARDIN L. Análise De Conteúdo. 3aㅗ reimp da 1aㅡ ed. São Paulo: Edições 70, 2016.

3. BEZERRA YRN, FEITOSA MZS. A afetividade do agente comunitário de saúde no território: um estudo com os mapas afetivos. Ciênc. saúde coletiva [online]. 2018, 23(3).

4. BONI V, QUARESMA SJ. Aprendendo a entrevistar: como fazer entrevistas em Ciências Sociais. Revista Eletrônica dos Pós-Graduandos em Sociologia Política da UFSC, 2005; 2(1): 68-80.

5. BRASIL CCP, et al. Community health workers caring for dependent elderly people. Ciência \& Saúde Coletiva, 2021; 26(1):109-118.

6. CAÇADOR BS, et al. Being a nurse in the family health strategy programme: challenges and possibilities. Reme : Rev. Min. Enferm. [online]. 2015 19(3).

7. DUBLAR C. A construção de si pela atividade de trabalho: a socialização profissional. Cadernos de Pesquisa 2012; 42(146): 351-367.

8. FERREIRA VS. Artes e manhas de entrevista compreensiva. Saúde Soc. São Paulo, 2014; 23(3): 979-992.

9. GUANAES-LORENZI C, PINHEIRO RL. Is the value of Community Healthcare Agents in Brazil's Family Health Strategy receiving full recognition? Ciência \& Saúde Coletiva, 2016; 21(8): 2537-2546.

10. JESUS AS, et al. Role of the community health agent: user's knowledge. Rev enferm UERJ, Rio de Janeiro, 2014; 22(2): 239-44.

11. MENEZES APR, et al. O futuro do SUS: impactos das reformas neoliberais na saúde pública - austeridade versus universalidade. Saúde Debate | Rio De Janeiro, 2019; 43(Especial 5): 58-70.

12. MINAYO MCDS, COSTA AP. Fundamentos Teóricos das Técnicas de Investigação Qualitativa. Rev Lusófona Educ [Internet]. 2018[cited 2019 Dec 18]; 40(40): 139-53.

13. MINISTÉRIO DA SAÚDE. OSUS de A a Z: garantindo saúde nos municípios/ Ministério da saúde, Conselho Nacional de Secretarias Municipais de Saúde; 3. Ed.; Brasília: Editora do Ministério da Saúde, Brasil, 2009.

14. MINISTÉRIO DA SAÚDE. Portaria nำ 2.436, de 21 de setembro de 2017. Aprova a Política Nacional de Atenção Básica, estabelecendo a revisão de diretrizes para a organização da Atenção Básica, no âmbito do Sistema Único de Saúde (SUS). Diário Oficial [da] República Federativa do Brasil. Brasil, 2017.

15. MOROSINI MV, FONSECA AF. Os agentes comunitários na Atenção Primária à Saúde no Brasil: inventário de conquistas e desafios. Saúde debate, Rio de Janeiro, 2018; 42(especial 1): 261-274.

16. NASCIMENTO VF, et al. Dificuldades apontadas pelo agente comunitário de saúde na realização do seu trabalho. Santa Maria, 2017; 43(1): 60-69.

17. PROETTI S. As pesquisas qualitativa e quantitativa como métodos de investigação científica: um estudo comparativo e objetivo. Revista Lumen. 2017; 2(4).

18. SAMUDIO JLP, et al. Agentes comunitários de saúde na atenção primária no brasil: multiplicidade de atividades e fragilização da formação. Trab. Educ. Saúde, Rio de Janeiro, 2017; 15(3): 745-770.

19. SANTOS AL, et al. Incorporação de Tecnologias de Informação e Comunicação e qualidade na atenção básica em saúde no Brasil. Cad. Saúde Pública 2017; 33(5).

20. SECCO AA, et al. Educação Permanente em Saúde para Agentes Comunitários: um Projeto de Promoção de Saúde. Gerais: Revista Interinstitucional de Psicologia, 2020; 13(1): e130108. 